\title{
DUALITY IN THE OPTIMAL CONTROL OF HYPERBOLIC EQUATIONS WITH POSITIVE CONTROLS
}

\author{
JONG YEOUL PARK and MI JIN LEE
}

(Received 11 September 1998 and in revised form 21 October 1998)

\begin{abstract}
We study the duality theory for hyperbolic equations. Also, we consider distributed control systems with positive control and convex cost functionals.

Keywords and phrases. Hyperbolic equations, duality theory, optimal control, convex cost function, positive control, distributed control systems.
\end{abstract}

2000 Mathematics Subject Classification. Primary 49K20, 49N15, 93C20.

1. Introduction. Recently Lions, motivated by various practical problems, started a program of studying the optimal control distributed systems. The developments of distributed systems are the establishing of the optimality systems which characterize the optimal control $[2,3,4,5]$. Duality theory for the corresponding parabolic equations with positive control has been given by Chan [1]. But, in this paper, we study the duality theory for hyperbolic distributed control systems. In fact, we consider distributed control systems with positive control and convex cost functionals. The approach presented exploits the fundamental results of Lions [2] on the optimality system which characterizes the optimal control. The method can be used to construct dual optimal systems when the controls are positive.

2. Duality in the optimal control hyperbolic equations with second-order operator. Let $\Omega$ be a bounded open set in $\mathbb{R}^{n}$ with smooth boundary $\Gamma$ and $Q=\Omega \times(0, T)$. The norm on $L^{2}(Q)$ is denoted by $|\cdot|$ and the corresponding inner product by $(\cdot, \cdot)$. In $Q$, information on the state is given by

$$
\begin{gathered}
\frac{\partial^{2} y}{\partial t^{2}}+\Delta y=u, \quad \text { in } Q, u \in U_{a d}, y \in L^{2}(Q), \\
y(0) \in K_{0}, \quad y=0, \quad \text { on } \sum
\end{gathered}
$$

where

$$
\begin{gathered}
U_{a d}=\left\{u \mid u \in L^{2}(Q), u \geq 0 \text { in } Q\right\}, \\
K_{0}=\left\{\phi \mid \phi \in H^{-1}(\Omega), \phi \geq 0 \text { in } \Omega\right\}, \\
\sum=\Gamma \times(0, T), \\
\Delta=\sum_{i=1}^{n} \frac{\partial^{2}}{\partial x_{i}^{2}} .
\end{gathered}
$$


Given $u \in U_{a d}$ and $y$ satisfying (2.1) we set

$$
J(y, u)=\frac{1}{2}\left|y-z_{d}\right|^{2}+\frac{1}{2}(N u, u),
$$

where $z_{d}$ is given in $L^{2}(Q)$ and $N>0$. The problem of optimal control is find inf $J(y, u)$, $u \in U_{a d}, u$ and $y$ being connected by (2.1). The above problem admits a unique solution $\{u, y\}$ which is characterized by the solution $\{u, y, p\}$ of the optimality system

$$
\begin{aligned}
& \frac{\partial^{2} y}{\partial t^{2}}+\Delta y=u \quad \text { for } u \geq 0, \\
& y=0 \quad \text { on } \sum, \\
& y(x, 0 ; u)=y_{0}(x), \quad \frac{\partial y}{\partial t}(x, 0 ; u)=y_{1}(x) \quad \text { in } \Omega, \\
& u \geq 0 \quad \text { a.e., in } Q, \\
& \frac{\partial^{2} p}{\partial t^{2}}+\Delta p=y-\mathscr{L}_{d} \quad \text { in } Q, \\
& p=0 \quad \text { on } \sum, \\
& p(T)=0, \quad \frac{\partial p}{\partial t}(T)=0 \quad \text { in } \Omega, \\
& p+N u \geq 0 \quad \text { in } Q, \\
& p(0) \geq 0, \\
& u(p+N u)=0, \\
& \frac{\partial p(0)}{\partial t} y(0)=0, \quad \frac{\partial y(0)}{\partial t} p(0)=0 \quad \text { in } \Omega .
\end{aligned}
$$

Next we prove the duality theorem.

THEOREM 1. Let $J=1 / 2\left|y-\mathscr{E}_{d}\right|^{2}+1 / 2(N u, u), K=-1 / 2|y|^{2}+1 / 2\left|\mathscr{E}_{d}\right|^{2}-1 / 2(N u$, $u)$. Assume $y_{0}, u_{0}, p_{0}$ satisfy $\left(\alpha_{1}\right),\left(\beta_{1}\right),\left(\gamma_{1}\right) ; y, u$ in $J$ satisfy $\left(\alpha_{1}\right)$; and $y, u$ in $K$ satisfy $\left(\beta_{1}\right)$. Then

$$
\inf _{\left(\alpha_{1}\right)} J=J\left(y_{0}, u_{0}\right)=K\left(y_{0}, u_{0}\right)=\sup _{\left(\beta_{1}\right)} K .
$$

Proof. (i) We begin by showing that $J=K$ at $\left(y_{0}, u_{0}, p_{0}\right)$.

$$
\begin{aligned}
J\left(y_{0}, u_{0}\right) & =J\left(y_{0}, u_{0}\right)-\left(u_{0}, p_{0}\right)-\left(u_{0}, N u_{0}\right) \\
& =J\left(y_{0}, u_{0}\right)-\left(y_{0}, \frac{\partial^{2} p_{0}}{\partial t^{2}}+\Delta p_{0}\right)-\left(u_{0}, N u_{0}\right)=K\left(y_{0}, u_{0}\right) .
\end{aligned}
$$

(ii) To show $\inf \left(\alpha_{1}\right) J=J\left(y_{0}, u_{0}\right)$, we must check that $J(y, u) \geq J\left(y_{0}, u_{0}\right)$, where $(y, u)$ satisfy $\left(\alpha_{1}\right)$ and $\left(y_{0}, u_{0}, p_{0}\right)$ satisfy $\left(\alpha_{1}\right),\left(\beta_{1}\right),\left(\gamma_{1}\right)$. Now, we have

$$
\begin{aligned}
J(y, u)-J\left(y_{0}, u_{0}\right) & \geq\left(y_{0}-z_{d}, y-y_{0}\right)+\left(N u_{0}, u-u_{0}\right) \\
& =\left(\frac{\partial^{2} p_{0}}{\partial t^{2}}+\Delta p_{0}, y-y_{0}\right)+\left(N u_{0}, u-u_{0}\right) \\
& =\left(p_{0}+N u_{0}, u-u_{0}\right) \geq 0 .
\end{aligned}
$$

Thus inf $J=J\left(y_{0}, u_{0}\right)$. 
(iii) To show $\sup _{\left(\beta_{1}\right)} K=K\left(y_{0}, u_{0}\right)$, we have to check that $K(y, u) \leq K\left(y_{0}, u_{0}\right)$, where $(y, u)$ satisfy $\left(\beta_{1}\right)$. But we have

$$
\begin{aligned}
J\left(y_{0}, u_{0}\right)-J(y, u) \geq & \left(y-z_{d}, y_{0}-y\right)+\left(N u, u_{0}-u\right)=\left(\frac{\partial^{2} p}{\partial t^{2}}+\Delta p, y_{0}-y\right) \\
& +\left(N u, u_{0}-u\right)+\left(\frac{\partial^{2} y_{0}}{\partial t^{2}}+\Delta y_{0}-u_{0}, p_{0}-p\right) \\
= & -\left(y-z_{d}, y\right)-(N u, u)+\left(y_{0}-z_{d}, y_{0}\right)+\left(N u_{0}, u_{0}\right) \\
& +\left(u_{0}, N u+p\right)-\left(u_{0}, p_{0}+N u_{0}\right) \\
\geq & -\left(y-z_{d}, y\right)-(N u, u)+\left(y_{0}-z_{d}, y_{0}\right)+\left(N u_{0}, u_{0}\right) .
\end{aligned}
$$

Therefore,

$$
J\left(y_{0}, u_{0}\right)-\left(y_{0}-z_{d}, y_{0}\right)-\left(N u_{0}, u_{0}\right) \geq J(y, u)-\left(y-z_{d}, y\right)-(N u, u),
$$

or

$$
K\left(y_{0}, u_{0}\right) \geq K(y, u)
$$

This completes the proof.

Now, we define the cost functional as

$$
J=\frac{1}{2}\left|y(T ; u)-z_{d}\right|_{L^{2}(\Omega)}^{2}+\frac{1}{2}(N u, u) .
$$

In $Q=\Omega \times(0, T)$, we consider the following system:

$$
\begin{aligned}
& \frac{\partial^{2} y}{\partial t^{2}}+\Delta y=u, \\
& y=0, \quad \text { on } \sum, \\
& y(x, 0 ; u)=y_{0}, \quad \frac{\partial y(x, 0 ; u)}{\partial t}=y_{1}, \\
& u \geq 0 \quad \text { a.e., in } Q, \\
& \frac{\partial^{2} p}{\partial t^{2}}+\Delta p=0 \quad \text { in } Q, \\
& p=0 \quad \text { on } \sum, \\
& p(x, T ; u)=0 \quad \text { for } x \in \Omega, \\
& \frac{\partial p(x, T ; u)}{\partial t}=y(x, T ; u)-z_{d} \quad \text { for } x \in \Omega, \\
& -p+N u \geq 0 \quad \text { in } Q, \\
& u(-p+N u)=0 \quad \text { a.e., in } Q, \\
& \frac{\partial p(0)}{\partial t} y(0)=0, \quad \frac{\partial y(0)}{\partial t} p(0)=0 \quad \text { on } \Omega .
\end{aligned}
$$


THEOREM 2. Let $J=1 / 2\left|y(T ; u)-z_{d}\right|_{L^{2}(\Omega)}^{2}+1 / 2(N u, u), K=-1 / 2|y(T ; u)|_{L^{2}(\Omega)}^{2}+$ $1 / 2\left|z_{d}\right|_{L^{2}(\Omega)}^{2}-1 / 2(N u, u)$. Assume $y_{0}, u_{0}, p_{0}$ satisfy $\left(\alpha_{2}\right),\left(\beta_{2}\right),\left(\gamma_{2}\right) ; y, u$ in J satisfy $\left(\alpha_{2}\right)$; and $y, u$ in $K$ satisfy $\left(\beta_{2}\right)$. Then

$$
\inf _{\left(\alpha_{2}\right)} J=J\left(y_{0}, u_{0}\right)=K\left(y_{0}, u_{0}\right)=\sup _{\left(\beta_{2}\right)} K .
$$

Proof. (i) Now we prove that $J=K$ at $\left(y_{0}, u_{0}, p_{0}\right)$. Then

$$
\begin{aligned}
J\left(y_{0}, u_{0}\right) & =J\left(y_{0}, u_{0}\right)+\left(p_{0}, u_{0}\right)-\left(N u_{0}, u_{0}\right) \\
& =J\left(y_{0}, u_{0}\right)+\left(\frac{\partial^{2} y_{0}}{\partial t^{2}}+\Delta y_{0}, p_{0}\right)-\left(N u_{0}, u_{0}\right) \\
& =-\frac{1}{2}\left|y_{0}(x, T ; u)\right|_{L^{2}(\Omega)}^{2}+\frac{1}{2}\left|z_{d}\right|_{L^{2}(\Omega)}^{2}-\frac{1}{2}\left(N u_{0}, u_{0}\right)=K\left(y_{0}, u_{0}\right)
\end{aligned}
$$

(ii) We show that $J(y, u) \geq J\left(y_{0}, u_{0}\right)$, where $(y, u)$ satisfy $\left(\alpha_{2}\right)$ and $\left(y_{0}, u_{0}, p_{0}\right)$ satisfy $\left(\alpha_{2}\right),\left(\beta_{2}\right),\left(\gamma_{2}\right)$.

$$
\begin{aligned}
J(y, u)-J\left(y_{0}, u_{0}\right) \geq & \left(y_{0}(T ; u)-z_{d}, y(T ; u)-y_{0}(T ; u)\right)_{L^{2}(\Omega)}+\left(N u_{0}, u-u_{0}\right) \\
= & \left(y_{0}(T ; u)-z_{d}, y(T ; u)-y_{0}(T ; u)\right)_{L^{2}(\Omega)}+\left(N u_{0}, u-u_{0}\right) \\
& -\left(\frac{\partial^{2} p_{0}}{\partial t^{2}}+\Delta p_{0}, y(t ; u)-y_{0}(t ; u)\right) \\
= & \left(-p_{0}+N u_{0}, u-u_{0}\right) \geq 0 .
\end{aligned}
$$

(iii) Now we claim that $\sup _{\left(\beta_{2}\right)} K=K\left(y_{0}, u_{0}\right)$.

$$
\begin{aligned}
J\left(y_{0}, u_{0}\right)-J(y, u) \geq & \left(y(T ; u)-z_{d}, y_{0}(T ; u)-y(T, u)\right)_{L^{2}(\Omega)}+\left(N u, u_{0}-u\right) \\
= & \left(y(T ; u)-z_{d}, y_{0}(T ; u)\right)_{L^{2}(\Omega)}-\left(y(T ; u)-z_{d}, y(T ; u)\right)_{L^{2}(\Omega)} \\
& +\left(N u, u_{0}\right)-(N u, u)-\left(\frac{\partial^{2} y_{0}}{\partial t^{2}}+\Delta y_{0}-u_{0}, p_{0}-p\right) \\
= & -\left(y(T ; u)-z_{d}, y(T ; u)\right)_{L^{2}(\Omega)}-(N u, u) \\
& +\left(y_{0}(T ; u)-z_{d}, y_{0}(T ; u)\right)_{l^{2}(\Omega)}+\left(N u_{0}, u_{0}\right) \\
& +\left(-p+N u, u_{0}\right)+\left(p_{0}-N u_{0}, u_{0}\right) .
\end{aligned}
$$

Therefore,

$$
\begin{aligned}
& J\left(y_{0}, u_{0}\right)-(\left.y_{0}(T ; u)-z_{d}, y_{0}(T ; u)\right)_{L^{2}(\Omega)}-\left(N u_{0}, u_{0}\right) \\
& \geq J(y, u)-\left(y(T ; u)-z_{d}, y(T ; u)\right)_{L^{2}(\Omega)}-(N u, u),
\end{aligned}
$$

and this implies

$$
\sup _{\left(\beta_{2}\right)} K(y, u)=K\left(y_{0}, u_{0}\right) .
$$

Thus, the proof is complete. 
3. Duality in the optimal control of hyperbolic equation with fourth-order operator. Let us consider the fourth-order differential operator.

$$
U_{a d}=\left\{u \mid u \in L^{2}(Q), u \geq 0 \text { in } Q\right\}, \quad \Delta=\sum_{i=1}^{n} \frac{\partial^{2}}{\partial x_{i}^{2}} .
$$

We consider a function $a(x, t)$ such that

$$
\left.\left.a \in C^{1}(] 0, T\right] ; L^{\infty}(\Omega)\right) .
$$

We introduce

$$
V=\left\{\phi \mid \phi, \Delta \phi \in L^{2}(\Omega)\right\}, \quad H=L^{2}(\Omega)
$$

and

$$
a(t ; \phi, \psi)=\int_{\Omega} a(x, t) \Delta \phi \Delta \psi d x, \quad \forall \phi, \psi \in V,
$$

given $u \in U_{a d}$ and we set

$$
J(y, u)=\frac{1}{2}\left|y-z_{d}\right|^{2}+\frac{1}{2}(N u, u),
$$

where $z_{d} \in L^{2}(Q), u \in U_{a d}$ and $N>0$.

$$
\begin{aligned}
& \frac{\partial^{2} y}{\partial t^{2}}+\Delta(a \Delta y)=u, \\
& \Delta y=0, \quad \frac{\partial \Delta}{\partial n} y=0, \quad \text { on } \sum \\
& \Delta y(x, 0 ; u)=y_{0}(x), \quad \frac{\partial y}{\partial t}(x, 0 ; u)=y_{1}(x), \quad x \in \Omega, \\
& u \geq 0, \quad \partial y(0) \geq 0, \quad \frac{\partial \Delta}{\partial n} y(0) \geq 0 . \\
& \frac{\partial p}{\partial t^{2}}+\Delta(a \Delta p)=y-z_{d} \quad \text { in } Q \\
& \Delta p=0, \quad \frac{\partial \Delta p}{\partial n}=0 \quad \text { on } \sum \\
& p(x, T ; u)=0, \quad \frac{\partial p(x, T ; u)}{\partial t}=0 \quad \text { on } \Omega \\
& p+N u \geq 0 \quad \text { in } Q . \\
& u(p+N u)=0, \quad \frac{\partial y(0)}{\partial t} p(0)=0 \quad \text { on } \Omega \\
& \frac{\partial p(0)}{\partial t} y(0)=0, \quad
\end{aligned}
$$

Now we claim the following.

THEOREM 3. Let $J=(1 / 2)\left|y-z_{d}\right|^{2}+(1 / 2)(N u, u), K=-(1 / 2)|y|^{2}+(1 / 2)\left|z_{d}\right|^{2}-$ $(1 / 2)(N u, u)$. Assume $y_{0}, u_{0}, p_{0}$ satisfy $\left(\alpha_{3}\right),\left(\beta_{3}\right),\left(\gamma_{3}\right) ; y, u$ in $J$ satisfy $\left(\alpha_{3}\right)$ and $y, u$ in $K$ satisfy $\left(\beta_{3}\right)$. Then

$$
\inf _{\left(\alpha_{3}\right)} J=J\left(y_{0}, u_{0}\right)=K\left(y_{0}, u_{0}\right)=\sup _{\left(\beta_{3}\right)} K .
$$


Proof. (i) We begin by showing that $J=K$ at $\left(y_{0}, u_{0}, p_{0}\right)$.

$$
\begin{aligned}
J\left(y_{0}, u_{0}\right) & =J\left(y_{0}, u_{0}\right)-\left(u_{0}, p_{0}\right)-\left(N u_{0}, u_{0}\right) \\
& =J\left(y_{0}, u_{0}\right)-\left(\frac{\partial^{2} y_{0}}{\partial t^{2}}+\Delta\left(a \Delta y_{0}\right), p_{0}\right)-\left(N u_{0}, u_{0}\right) \\
& =J\left(y_{0}, u_{0}\right)-\left(y_{0}, y_{0}-z_{d}\right)-\left(N u_{0}, u_{0}\right)=K\left(y_{0}, u_{0}\right) .
\end{aligned}
$$

(ii) We show that $J(y, u) \geq J\left(y_{0}, u_{0}\right)$.

$$
\begin{aligned}
J(y, u)-J\left(y_{0}, u_{0}\right) \geq & \left(y_{0}-z_{d}, y-y_{0}\right)+\left(N u_{0}, u-u_{0}\right) \\
= & \left(\frac{\partial^{2} p_{0}}{\partial t^{2}}+\Delta\left(a \Delta p_{0}\right), y-y_{0}\right)+\left(N u_{0}, u-u_{0}\right) \\
& +\left(N u_{0}, u-u_{0}\right)=\left(p_{0}+N u_{0}, u-u_{0}\right) \geq 0
\end{aligned}
$$

(iii) We prove that $K(y, u) \leq K\left(y_{0}, u_{0}\right)$.

$$
\begin{aligned}
J\left(y_{0}, u_{0}\right)-J(y, u) \geq & \left(y-z_{d}, y_{0}-y\right)+\left(N u, u_{0}-u\right) \\
= & \left(\frac{\partial^{2} p}{\partial t^{2}}+\Delta(a \Delta P), y_{0}-y\right)+\left(N u, u_{0}-u\right) \\
& +\left(\frac{\partial^{2} y_{0}}{\partial t^{2}}+\Delta\left(a \Delta y_{0}\right)-u_{0}, p_{0}-p\right) \\
= & -\left(y-z_{d}, y\right)+\left(y_{0}-z_{d}, y_{0}\right)+\left(p+N u, u_{0}\right)-\left(N u_{0}+p_{0}, u_{0}\right) \\
& -(N u, u)+\left(N u_{0}, u_{0}\right)+\left(u_{0}, p\right)-\left(y-\mathscr{L}_{d}, y_{0}\right) \\
\geq & -\left(y-z_{d}, y\right)+\left(y_{0}-z_{0}, y_{0}\right)-(N u, u)+\left(N u_{0}, u_{0}\right) .
\end{aligned}
$$

Therefore,

$$
K\left(y_{0}, u_{0}\right) \geq K(y, u)
$$

Now, we set the following cost function:

$$
J=\frac{1}{2}\left|y(T ; u)-z_{d}\right|_{L^{2}(\Omega)}^{2}+\frac{1}{2}(N u, u),
$$

where $z_{d} \in L^{2}(Q), u \in U_{a d}$ and $N>0$ and associated following systems:

$$
\begin{aligned}
& \frac{\partial^{2} y}{\partial t^{2}}+\Delta(a \Delta y)=u, \\
& \Delta y=0, \quad \frac{\partial \Delta}{\partial n} y=0 \quad \text { on } \sum \\
& \Delta y(x, 0 ; u)=y_{0}(x), \quad \frac{\partial y}{\partial t}(x, 0 ; u)=y_{1}(x), \quad x \in \Omega, \\
& u \geq 0, \quad \partial y(0) \geq 0, \quad \frac{\partial \Delta}{\partial n} y(0) \geq 0 .
\end{aligned}
$$




$$
\begin{aligned}
& \frac{\partial^{2} p}{\partial t^{2}}+\Delta(a \Delta p)=0 \quad \text { in } Q, \\
& \Delta p=0, \quad \frac{\partial \Delta}{\partial n} p=0 \quad \text { on } \sum \\
& p(x, T ; u)=0, \quad \frac{\partial p}{\partial t}(x, T ; u)=y(x, T ; u)-z_{d} \quad \text { on } \Omega, \\
& -p+N u \geq 0 \quad \text { in } Q, \\
& u(p+N u)=0, \quad \frac{\partial y(0)}{\partial t} p(0)=0 \quad \text { on } \Omega . \\
& \frac{\partial p(0)}{\partial t} y(0)=0, \quad
\end{aligned}
$$

we have the duality result.

THEOREM 4. Let $J=(1 / 2)\left|y(T ; u)-z_{d}\right|_{L^{2}(\Omega)}^{2}+(1 / 2)(N u, u), K=-(1 / 2)|y(T ; u)|_{L^{2}(\Omega)}^{2}$ $+(1 / 2)\left|z_{d}\right|_{L^{2}(\Omega)}^{2}-(1 / 2)(N u, u)$. Assume $y_{0}, u_{0}, p_{0}$ satisfy $\left(\alpha_{4}\right),\left(\beta_{4}\right),\left(\gamma_{4}\right) ; y, u$ in $J$ satisfy $\left(\alpha_{4}\right)$ and $y, u$ in $K$ satisfy $\left(\beta_{4}\right)$. Then

$$
\inf _{\left(\alpha_{4}\right)} J=J\left(y_{0}, u_{0}\right)=K\left(y_{0}, u_{0}\right)=\sup _{\left(\beta_{4}\right)} K
$$

Proof. (i) We begin to prove that

$$
\begin{aligned}
J\left(y_{0}, u_{0}\right)= & J\left(y_{0}, u_{0}\right)-\left(u_{0}, p_{0}\right)-\left(N u_{0}, u_{0}\right) \\
= & J\left(y_{0}, u_{0}\right)-\left(\frac{\partial^{2} y_{0}}{\partial t^{2}}+\Delta\left(a \Delta y_{0}\right), p_{0}\right)-\left(N u_{0}, u_{0}\right) \\
= & J\left(y_{0}, u_{0}\right)-\left(y_{0}(T ; u)-z_{d}, y_{0}(T ; u)\right)_{L^{2}(\Omega)} \\
& +\left(y_{0}, \frac{\partial^{2} p_{0}}{\partial t^{2}}+\Delta\left(a \Delta p_{0}\right)\right)-\left(N u_{0}, u_{0}\right) \\
= & -\frac{1}{2}\left|y_{0}(T ; u)\right|_{L^{2}(\Omega)}^{2}+\frac{1}{2}\left|z_{d}\right|_{L^{2}(\Omega)}^{2}-\frac{1}{2}\left(N u_{0}, u_{0}\right)=K\left(y_{0}, u_{0}\right)
\end{aligned}
$$

(ii) We claim that $J(y, u) \geq J\left(y_{0}, u_{0}\right)$.

$$
\begin{aligned}
J(y, u)-J\left(y_{0}, u_{0}\right) \geq & \left(y_{0}(T ; u)-z_{d}, y(T ; u)-y_{0}(T ; u)\right)_{L^{2}(\Omega)}+\left(N u_{0}, u-u_{0}\right) \\
= & \left(y_{0}(T ; u)-z_{d}, y(T ; u)-y_{0}(T ; u)\right)_{L^{2}(\Omega)}+\left(N u_{0}, u-u_{0}\right) \\
& -\left(\frac{\partial^{2} p_{0}}{\partial t^{2}}+\Delta\left(a \Delta p_{0}\right), y(t ; u)-y_{0}(t ; u)\right) \\
= & \left(-p+N u_{0}, u-u_{0}\right) \geq 0 .
\end{aligned}
$$

(iii) Now, we verify that $K(y, u) \leq K\left(y_{0}, u_{0}\right)$.

$$
\begin{aligned}
J\left(y_{0}, u_{0}\right)-J(y, u) & \geq\left(y(T ; u)-z_{d}, y_{0}(T ; u)-y(T ; u)\right)_{L^{2}(\Omega)}+\left(N u, u_{0}-u\right) \\
& =\left(y(T ; u)-z_{d}, y_{0}(T ; u)\right)_{L^{2}(\Omega)}-\left(y(T ; u)-z_{d}, y(T ; u)\right)_{L^{2}(\Omega)}
\end{aligned}
$$




$$
\begin{aligned}
& +\left(N u, u_{0}\right)-(N u, u)-\left(\frac{\partial^{2} y_{0}}{\partial t^{2}}+\Delta\left(a \Delta y_{0}\right)-u_{0}, p_{0}-p\right) \\
= & -\left(y(T ; u)-z_{d}, y(T ; u)\right)_{L^{2}(\Omega)}-(N u, u) \\
& +\left(y_{0}(T ; u)-z_{d}, y_{0}(T ; u)\right)_{L^{2}(\Omega)}+\left(N u_{0}, u_{0}\right) \\
& +\left(-p+N u, u_{0}\right)+\left(p_{0}-N u_{0}, u_{0}\right) .
\end{aligned}
$$

This implies that

$$
\sup _{\left(\beta_{4}\right)} K=K\left(y_{0}, u_{0}\right)
$$

ACKNOWLEDGEMENT. This work was supported by KOSEF, 1996.

\section{REFERENCES}

[1] W. L. Chan, Duality in the optimal control of non-well-posed parabolic equations with positive controls, J. Math. Anal. Appl. 107 (1985), no. 2, 509-519. MR 86f:49048. Zbl 593.49009.

[2] J. L. Lions, Optimal Control of Systems Governed by Partial Differential Equations, Die Grundlehren der mathematischen Wissenschaften, vol. 170, Springer-Verlag, New York, Berlin, 1971. MR 42\#6395. Zbl 203.09001.

[3] _ Optimal control of non-well-posed distributed systems and related nonlinear partial differential equations, Nonlinear problems: present and future (Amsterdam, New York), North-Holland Math. Stud., vol. 61, North-Holland, 1982, pp. 3-16. MR 84d:49020. Zbl 495.49013.

[4] _ Optimal control of unstable distributed systems, Current problems in numerical and applied mathematics (Novosibirsk), "Nauka" Sibirsk. Otdel., 1983, pp. 7-19. MR 86b:49009. Zbl 547.49012.

[5] J. Mossino, An application of duality to distributed optimal control problems with constraints on the control and the state, J. Math. Anal. Appl. 50 (1975), 223-242. MR 52 6530. Zbl 304.49003.

PARK: DePartment of MATHematics, Pusan National University, PUSAN 609-735, KoReA E-mail address: jyepark@hyowon. pusan.ac. kr

LeE: Department of Mathematics, Pusan National University, Pusan 609-735, Korea 


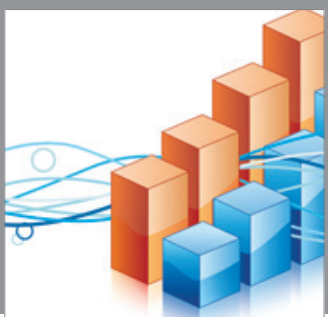

Advances in

Operations Research

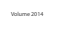

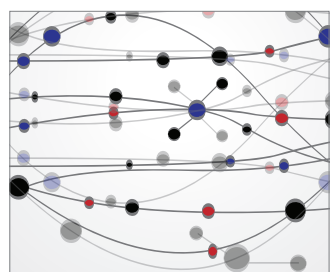

\section{The Scientific} World Journal
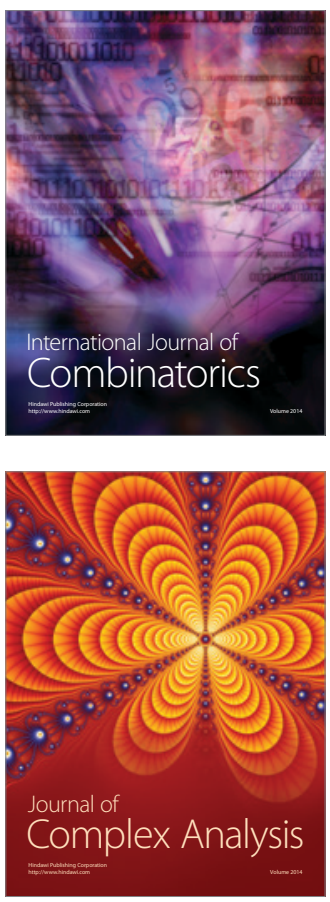

International Journal of

Mathematics and

Mathematical

Sciences
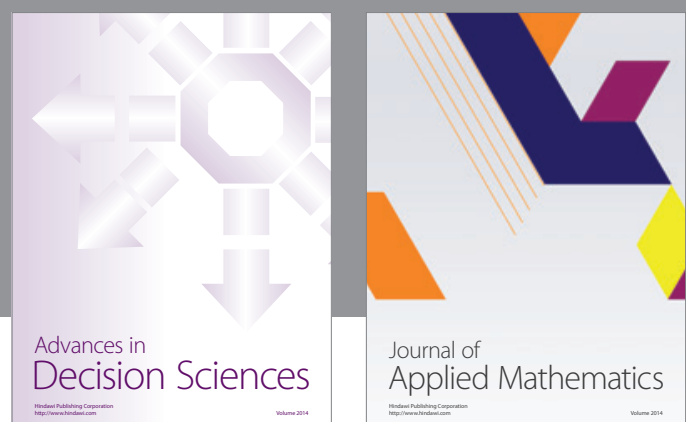

Journal of

Applied Mathematics
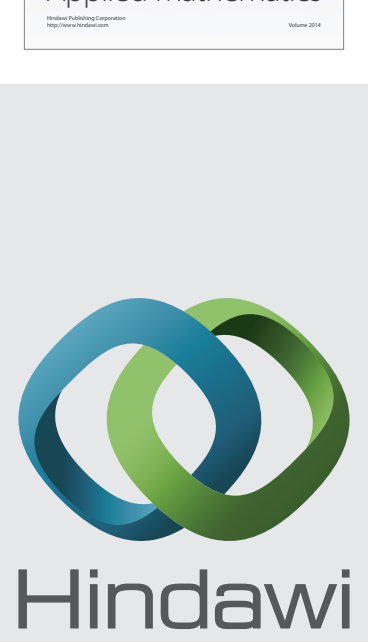

Submit your manuscripts at http://www.hindawi.com
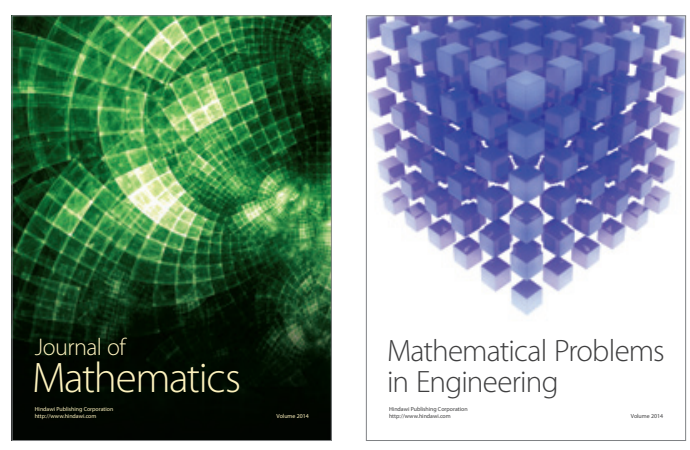

Mathematical Problems in Engineering
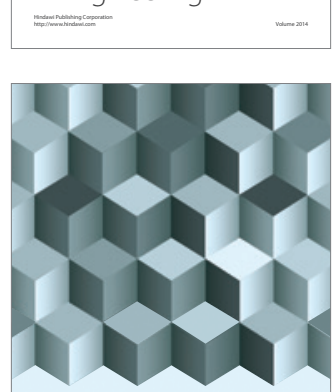

Journal of

Function Spaces
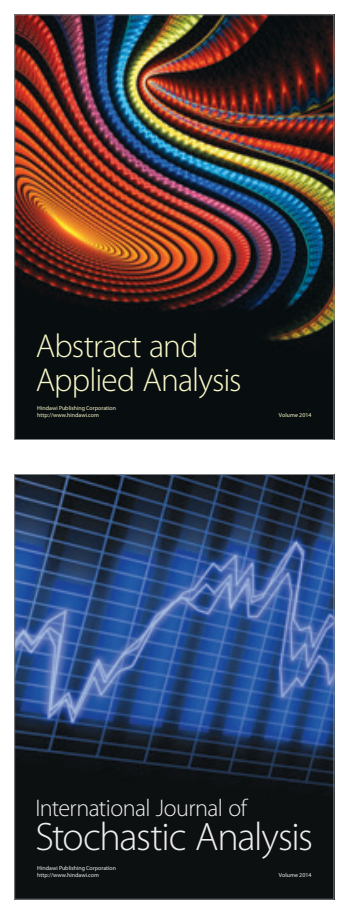

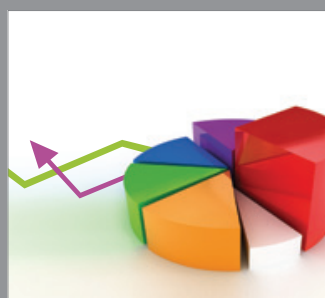

ournal of

Probability and Statistics

Promensencen
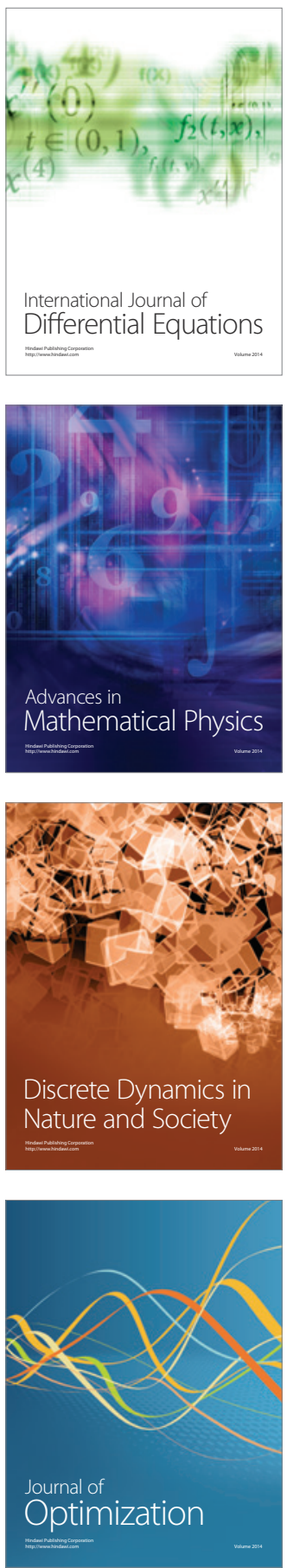